\title{
Preface to the special issue on recent advances of deep structure, seismic anisotropy and seismotectonics in China*
}

\author{
Dongping $\mathrm{Wei}^{1}$ Fenglin $\mathrm{Niu}^{2} \quad \mathrm{Yuan}_{\mathrm{Gao}}^{3, \star}$ and Xuzhang Shen ${ }^{4}$ \\ ${ }^{1}$ College of Earth Science, Graduate University of Chinese Academy of Sciences, Beijing 100049, China \\ ${ }^{2}$ Department of Earth Science, Rice University, Houston, TX 77005, USA \\ ${ }^{3}$ Institute of Earthquake Science, China Earthquake Administration, Beijing 100036, China \\ ${ }^{4}$ Lanzhou Institute of Seismology, China Earthquake Administration, Lanzhou 730000, China
}

A workshop on crustal structure and seismotectonics was held on the Chinese Teacher's Day, the September 10th of 2011, in the city Lanzhou, China. Scientists and graduate students from Chinese Academy of Sciences, China Earthquake Administration, Chinese Academy of Geological Sciences, and Japan Agency for Marine-Earth Science and Technology delivered over 20 oral presentations, with topics covering crustal and upper mantle structure, seismic anisotropy, recent earthquakes and seismotectonics, and earthquake predictions. The presentations were followed by an intensive discussion on fundamental processes in shaping the Chinese mainland as well as in nucleating earthquakes. As a keynote speaker of the workshop, professor Huilan Zhou from the Graduate University of Chinese Academy of Sciences was invited to give a summary talk at the end of the workshop. Although retired, professor Zhou delivered an extraordinary presentation that summarized not only her passion on seismology over the last forty years, but also rendered future directions in exploring earthquake physics and Earth's interior in the digital era of seismology.

Seismology is traditionally divided to earthquake seismology and structural seismology. Chinese seismologists have been making tremendous contributions to both fields. Some of Chinese seismologists were particularly well recognized by their works on earthquake physics in 1980s. For examples, Chen and Knopoff (1986a, b) are two classic papers that provided profound

\footnotetext{
* Received 20 January 2012; accepted in revised form 24 January 2012; published 10 February 2012.

+ Corresponding author. e-mail: gaoyuan@seis.ac.cn

(c) The Seismological Society of China, Institute of Geophysics, China Earthquake Administration, and Springer-Verlag Berlin Heidelberg 2012
}

insights on the dynamics of shear cracks. Zhou et al. (1983a, b), on the other hand, are typically among the early several studies on rupture complexity of intraplate large earthquakes in China. While modern seismology continues evolving with many exciting discoveries, such as the finding of episodic tremor in young subduction zone, the observational nature of seismology has never been changed. The Chinese seismological society has caught world's attention once again by the completion of the largest broadband seismic network on the globe. This has been providing an unprecedented opportunity for the new generation of seismologists in studying the physics of both Earth's interior and earthquakes. Many of the studies presented at the workshop are built upon this extraordinary seismic network.

Professor Keiichi Aki, the former president of the Seismological Society of America, once said that "the joy of being a seismologist comes to you, when you find something new about the earth's interior from the observation of seismic waves obtained on the surface, and realize that you did it without penetrating the earth or touching or examining it directly". The central role of seismology in solid Earth science in exploring Earth's interior was well described in two classic textbooks, Principle of Seismology (Xu and Zhou, 1982) and Physics of Earth's Interior (Zhou, 1990). These two books have been widely used in high-level geophysics courses, and have nurtured several generations of undergraduate and graduate students in Chinese Academy of Sciences. Seismologists and geophysicists who once worked or studied in Graduate University of Chinese Academy of Sciences have conducted creative works in numerous fields, special in characterizing the seismic source of large earthquakes in China (such as Zhou et 
al., 1983a, b), investigating the crustal stress by inversion of nodal-plane data (Zhou and Ding, 1995), analyzing seismicity in typical tectonic zone combining with characteristics of strong earthquakes (such as Gao et al., 2000), exploring the Earth's deep structure by receiver functions and Rayleigh wave dispersion (such as Shen et al., 2008; Ma and Zhou, 2007) or by ray tracing technique (such as Lei et al., 2002) as well as in studying theoretical travel time of seismic phases at the upper mantle (Wei et al., 1993), also in measuring, calculating and interpreting seismic anisotropy (such as Gao et al., 1998; Liu et al., 1998), which have led to many significant discoveries in these fields in seismology.

Following the workshop, we planned a special issue on deep structure, seismic anisotropy and seismotectonics presented in the workshop. We selected a total of 12 papers that cover exciting results from receiver functions images of crustal and mantle structure by discussions on double-crust structure, Conrad discontinuity, the accuracy and stability with stacking technique as well as usage of a peeling linear inversion; modeling of crustal attenuation around a fault; distinct layering at the top of the outer core by PKKP waves; measurements of seismic anisotropy across China, both in continental and regional scales, in order to searching for connection with stress and faults; novel techniques to construct synthetic seismograms and their receiver functions; double seismic zones in subduction zones; tectonic implications and consequences of two recent strong earthquakes in Chinese mainland, i.e., the $2010 M_{\mathrm{S}} 7.1$ Yushu earthquake and the $2011 M_{\mathrm{S}} 5.8$ Yingjiang earthquake; and discussions on equivalence of major propagator algorithms. The diversity of the papers presented here shows the rapid progress in seismology and geodynamics in recent years in China.

We would like to thank all the authors for their timely contributions to this special issue. Also, with common wish from all the authors of this special issue, we would express our sincere and heartfelt gratitude to Prof. Zhou's education in the past, as well as in appreciation to her long-term contribution to the seismology and geodynamics community over the last 40 years. We appreciate the reviewers for their careful and constructive comments to improve papers, thank Dr. Lili Tian and Ms. Jiangli Liu in the editorial office of Earthquake Science with their help in producing this special issue. Finally, we thank Lanzhou Institute of Seismology, China Earthquake Administration, for organizing the workshop and partly support by basic research project of Institute of Earthquake Science, China Earthquake Ad- ministration (No. 2011IESLZ05 and No. 2009-11\&21).

\section{References}

Chen Y T and Knopoff L (1986a). Static shear crack with a zone of slip-weakening. Geophys $J R$ astr Soc 87(3): $1005-1024$.

Chen Y T and Knopoff L (1986b). The quasistatic extension of a shear crack in a viscoelastic medium. Geophys $J R$ astr Soc 87(3): 1 025-1 039.

Gao Y, Wang P D, Zheng S H, Wang M, Chen Y T and Zhou H L (1998). Temporal changes in shear-wave splitting at an isolated swarm of small earthquakes in 1992 near Dongfang, Hainan Island, southern China. Geophys J Int 135: 102-112, doi:10.1046/j.1365-246X.1998.00606.x

Gao Y, Wu Z L, Liu Z and Zhou H L (2000). Seismic source characteristics of nine strong earthquakes from 1988 to 1990 and earthquake activity since 1970 in the SichuanQinghai-Xizang (Tibet) zone of China. Pure Appl Geophys 157(9): 1 423-1 443.

Lei J S, Zhou H L and Zhao D P (2002). 3-D velocity structure of P-wave in the crust and upper-mantle beneath Pamir and adjacent region. Chinese J Geophys 45(6): 802-811 (in Chinese with English abstract).

Liu X Q, Zhou H L, Zheng Z Z, Gao Y, Li H, Ma Y L and Liang K L (1998). The characteristics of shear-wave splitting parameters in the presences of two weak azimuth anisotropic layers. Chinese J Geophys 41(5): 680690 (in Chinese with English abstract).

Ma Y L and Zhou H L (2007). Crustal thicknesses and Poisson's ratios in China by joint analysis of teleseismic receiver functions and Rayleigh wave dispersion. Geophys Res Lett 34: L12304, doi:10.1029/2007GL029848.

Shen X Z, Zhou H L and Kawakatsu H (2008). Mapping the upper mantle discontinuities beneath China with teleseismic receiver functions. Earth Planets Space 60(7): 713-719.

Wei D P, Zhou H L and Wang Z Q (1993). Travel time of related seismic phases at $670 \mathrm{~km}$ discontinuity at the upper mantle. Chinese Science Bulletin 38(22): 2 063-2 066 (in Chinese).

Xu G M and Zhou H L (1982). Principles of Seismology. Science Press, Beijing, 445pp (in Chinese).

Zhou H L (1990). Physics of Earth's Interior. Seismological Press, Beijing, 314pp (in Chinese).

Zhou H L and Ding R G (1995). Study of the characteristics of crustal stress field in East China by inversion of nodal-plane data. Tectonophysics 245: 53-59.

Zhou H L, Liu S and Kanamori H (1983a). Source processes of large earthquakes along the Xianshuihe fault in southwestern China. Bull Seismol Soc Am 73(2): 537-551.

Zhou H L, Allen C R and Kanamori H (1983b). Rupture complexity of 1970 Tonghai and Luhuo earthquakes, China, from P-wave inversion, and relationship to surface faulting. Bull Seismol Soc Am 73(6): 1 585-1 597. 